\title{
COMPUTING THE EULER CHARACTERISTIC OF A MANIFOLD WITH BOUNDARY
}

\author{
HAROLD LEVINE
}

(Communicated by Ronald Stern)

\begin{abstract}
Given a stable map, $f$, from an even-dimensional, compact manifold with boundary, $M$, into $\mathbb{R}^{2}$, the Euler characteristic of $M, \chi(M)$, is expressed in terms of projectivized rotation numbers of $f$ restricted to $S(f)$, the singular curves of $f$, and of $f$ restricted to $S(f \mid \partial M)$, the singular curves of $f$ restricted to the boundary of $M$.
\end{abstract}

Let $M$ be a compact, even-dimensional manifold and let $f$ be a smooth map from $M$ into $\mathbb{R}^{2}$. In [1], assuming the local stability of $f$, a formula for $\chi(M)$, the Euler characteristic of $M$ is given in terms of the singularities of $f$. This result is very much in the spirit of the formula of Morse for $\chi(M)$ and is in a real way a corollary of that result. The object of this note is to extend the result of [1] to manifolds with boundary. In the special case where $M$ is 2-dimensional, this result has been applied to questions in gravitational lensing in astrophysics, the results of which will appear in joint work with Arlie Petters and Joachim Wambsganss [0].

Let $M$ be a compact, $n$-dimensional manifold, $n \geq 2$, and let $f: M \rightarrow \mathbb{R}^{2}$ be a smooth mapping about which we make the following assumptions:

$$
\left\{\begin{array}{l}
J^{1}(f) \text { is transverse to both } S_{1}\left(M, \mathbb{R}^{2}\right) \text { and to } S_{2}\left(M, \mathbb{R}^{2}\right), \\
J^{2}(f) \text { is transverse to } S_{1}^{2}\left(M, \mathbb{R}^{2}\right) .
\end{array}\right.
$$

These assumptions translate into local restrictions on $f$, namely:

(i) The rank of $T f$, rk $T f \geq 1$.

(ii) At each point, $P$, of $S_{1}(f)=\{Q \in M \mid \operatorname{rk} T f=1\}$, we can choose coordinates, $\left\{u, x, z_{1}, \ldots, z_{n-2}\right\}$, centered at $P$ and $\{U, Y\}$, centered at $f(P)$ such that $f$ has one of the forms:

$$
\left\{\begin{array}{l}
U \circ f(u, x, z)=u, \\
Y \circ f(u, x, z)=\sum_{i=1}^{n-2} \pm z_{i}^{2} \pm x^{2}=\mathscr{Q}(z, x), \\
\quad \text { a nonsingular quadratic form in } x \text { and the } z \text { 's, }
\end{array}\right.
$$

Received by the editors October 1, 1993.

1991 Mathematics Subject Classification. Primary 57R45, 58C27; Secondary 57R35, 58C25.

Key words and phrases. Stable map, singular curve, fold point, cusp point, rotation number, projectivized rotation number, Euler characteristic, manifold with boundary. 
or

$$
\left\{\begin{array}{l}
U \circ f(u, x, z)=u, \\
Y \circ f(u, x, z)=\sum_{i=1}^{n-2} \pm z_{i}^{2} \pm x u \pm x^{3}
\end{array}\right.
$$

These local restrictions imply, in turn, that the singular set of $f, S(f)=$ $S_{1}(f)$, consists of smooth, nonintersecting closed curves and that on those curves there is a discrete set of cusp points, $S_{1}^{2}(f)$. If $c$ is a component of $S(f), f \mid c$ is an "immersion-with cusps"; that is, at each of a finite set of "cusp" points of $c, S_{1}^{2}(f) \cap c, f$ has one of the forms (2) and $f \mid c$ is singular. At all other points of $c$, the "fold" points of $c, f$ has one of the forms (1) and $f \mid c$ is an immersion.

Let $P\left(\mathbb{R}^{2}\right)$ be the real projective line, the space of lines through 0 in $\mathbb{R}^{2}$. Considering the mapping, $\phi: S(f)-S_{1}^{2}(f) \rightarrow P\left(\mathbb{R}^{2}\right)$, which takes a fold point, $P$, to the line through 0 in $\mathbb{R}^{2}$, parallel to the tangent line to the $f$-image of $S(f)$ at $f(P)$. It is shown in [1] that $\phi$ extends to a smooth map of all of $S(f)$ into $P\left(\mathbb{R}^{2}\right)$. If $c$ is an oriented component of $S(f)$, we denote by $r(c)$, the degree of $\phi \mid c$. We sometimes speak of $r(c)$ as the projectivized rotation number of $c$. As usual, for an immersion, $\alpha: S^{1} \rightarrow \mathbb{R}^{2}$, for $S^{1}$ oriented, we denote by $\operatorname{rot}(\alpha)$, the rotation number of $\alpha$, the degree of the map $S^{1} \rightarrow S^{1}: t \rightarrow \frac{\alpha^{\prime}(t)}{\left|\alpha^{\prime}(t)\right|}$.

Theorem [1]. Let $M$ be an even-dimensional, compact manifold and let $f: M \rightarrow$ $\mathbb{R}^{2}$ satisfy conditions $(\star)$. There is a unique orientation of $S(f)$ such that:

$$
\chi(M)=\sum_{c} r(c)
$$

where the sum runs over all the components of $S(f)$ and $\chi(M)$ is the Euler characteristic of $M$.

Notes. (1) The theorem as stated in [1] requires $M$ to be oriented however the restriction is irrelevant to the proof given there.

(2) The orientation referred to in the theorem is the one that makes all of the indices of the quadratic forms, $\mathscr{Q}(z, x)$, in (1) even. See [1] for details.

The object of this note is to extend this theorem to compact, even-dimensional manifolds with boundary. In order to have control on the boundary as well as in the interior of $M$ we strengthen the assumption on $f$ as follows:

We assume that $f: M \rightarrow R^{2}$ is stable with singular set, $S(f)$, disjoint from the boundary of $M, \partial M$.

The assumption that $f$ is stable implies that $f$ satisfies condition $(\star)$. In addition it guarantees that the only double points of $f \mid S(f)$ are fold points and that the crossings of the images are normal. However the reason for making this assumption is:

Lemma. Let $M$ and $P$ be manifolds, $M$ compact with boundary; and let $f: M \rightarrow P$ be stable. Then $f \mid \partial M: \partial M \rightarrow P$ is also stable.

Proof. If $\phi$ is a diffeomorphism of $M$ with itself, $\phi \mid \partial M$ is also a diffeomorphism of $\partial M$ with itself. Thus, if $\phi$ and $\psi$ are diffeomorphisms of $M$ and $P$ respectively and if $\tilde{f}=\psi \circ f \circ \phi$, then $\tilde{f}|\partial M=\psi \circ f \circ \phi| \partial M=\psi \circ f|\partial M \circ \phi| \partial M$. That is, $\tilde{f} \mid \partial M$ is equivalent to $f \mid \partial M$.

The question remains if any mapping, $g$, of $\partial M$ into $N$, close to $f \mid \partial M$, is equivalent to $f \mid \partial M$. Or equivalently, if $f \mid \partial M$ is stable. But that is clear 
since the stability of $f \mid \partial M$ is specified by open multijet conditions at one, two, or three points of $\partial M$. If the stability conditions for $f \mid \partial M$ were violated at some multijet, then $f$ itself could be altered in a neighborhood (nbhd) of one, two, or three points of $\partial M$ to obtain $\tilde{f}$ near $f$ such that $\tilde{f} \mid \partial M$ is not equivalent to $f \mid \partial M$.

To compute $\chi(M)$, we double $M$, obtaining $\widehat{M}$. The manifold, $\widehat{M}$, is constructed from $M$ by taking the quotient of $M \times\{-1,1\}$ by the relation $(x, t) \sim\left(x^{\prime}, t^{\prime}\right)$ iff $x=x^{\prime}$ and either $t=t^{\prime}$ or $x \in \partial M$. For convenience, we let $N=\partial M$. The differentiable structure that we give to $\widehat{M}$ is easy to describe. We declare as diffeomorphisms the maps $[M, \pm 1] \rightarrow M:[x, \pm 1] \rightarrow$ $x$. Finally we declare as diffeomorphism a map of $N \times(-1,1)$ onto a nbhd of the image of $N$ in $\widehat{M}$ as follows: Since the normal bundle of $N$ is a trivial line bundle over $N$, there is a diffeomorphism, $\phi$, of $N \times[0,1)$, with a nbhd, $V$, of $N$ in $M$. It is no restriction to assume that for $x \in N$, $\phi(x, 0)=x$. Let $\widehat{V}=(V \times\{-1,1\}) / \sim$ and declare, as diffeomorphism, the map $\psi: N \times(-1,1) \rightarrow \widehat{V}$, by $\psi(x, s)=\left[\phi(x,|s|), \frac{s}{\mid s]}\right]$, for $s \neq 0$, and $\psi(x, 0)=[x, \pm 1]$. These three diffeomorphisms give the differentiable structure on $M$ compatible with that of two copies of $M$ as a submanifold with boundary of $\widehat{M}$.

We define the mapping $\hat{f}: \widehat{M} \rightarrow \mathbb{R}^{2}$ "essentially" as follows: $\hat{f}([x, t])=$ $f(x)$. The "essentially" means that this definition is precise except in the double collar nbhd of $\partial M$ where the fold along $\partial M$ is smoothed into a stable fold. We clarify this construction.

By our assumption, $S(f \mid N)$ consists of nonsingular, noninteresting, closed curves which are "immersed with cusps" by $f$ into $\mathbb{R}^{2}$. Since we assume that $S(f) \| N$, we know that for $x \in S(f \mid N), \operatorname{ker}\left(T f_{x}\right) \subseteq T N_{x}$. Thus $T f$ always has rank one on the space normal to $N$ at $x$. We now proceed to define $\hat{f}$ :

Let $g:(-1,1) \rightarrow[0,1)$ be a smooth, even function such that for $0 \leq|s|<$ $\varepsilon<\frac{1}{2}, g(s)=s^{2}$, and for $|s|>(1-\varepsilon), g(s)=|s|$ and for $s \neq 0, s g^{\prime}(0)>0$.

Let $\hat{f}: \widehat{M} \rightarrow \mathbb{R}^{2}$ be defined by $\hat{f}([x, t])=f(x)$, for $x \notin \phi(N \times[0,1-\varepsilon])$, and $\hat{f}([\phi(x, s), t])=f\left(\phi(x, g(s))\right.$, for $(x, s) \in N \times\left[0,1-\frac{\varepsilon}{2}\right)$. In particular, for $(x, s) \in N \times(-\varepsilon, \varepsilon), \hat{f}(\psi(x, s))=f\left(\phi\left(x, s^{2}\right)\right)$.

Lemma. The map $\hat{f}: \widehat{M} \rightarrow \mathbb{R}^{2}$ defined above satisfies condition $(\star)$.

Note. The reason that we cannot conclude that $\hat{f}$ is stable is that each curve, $c$, of $S(f)$ appears as a pair of curves, $[c, 1]$ and $[c,-1]$ in $S(\hat{f})$. For each point, $x \in c, \hat{f}[x, \pm 1]=f(x)$, hence the mappings, $\hat{f} \mid[c, 1]$ and $\hat{f} \mid[c,-1]$, violate the normal crossing requirement for the singular image curves of a stable map. Further, the singularities of $f \mid \partial M, S(f \mid \partial M)$, appear among singularities of $\hat{f}$. The assumption on $f$ does not guarantee that the curves which are the $f$-image of $S(f)$ and $(f \mid \partial M)$-image of $S(f \mid \partial M)$ are in general position as would be required by the stability of $\hat{f}$.

Proof. We need only check the condition $(\star)$ at points of $[S(f \mid \partial M), \pm 1]$. Since the normal forms (1) and (2) are determined by the 2- and 3-jets of the mappings respectively, our proof is very easy. A germ of a map, $g$, at a point 
$Q$ in a manifold, $A$, to a manifold $B$, we denote by $g:(A, Q)-\rightarrow B$, or simply by $g: A-\rightarrow B$ when the point $Q$ is understood.

Let $P$ be a point of $S(f \mid N)$. Choose coordinate map germ, $\theta: \mathbb{R}^{n-1}-\rightarrow N$ centered at $P,\{u, x, z\}$, and $\{U, Y\}$ for $\mathbb{R}^{2}$ centered at $f(P)$ such that in terms of these coordinates $f \mid N$ has one of the forms, (1) or (2). We denote either of these as:

$$
\left\{\begin{array}{l}
U(f(\phi(\theta(u, x, z), 0)))=u \\
Y(f(\phi(\theta(u, x, z), 0)))=G(u, x, z),
\end{array}\right.
$$

where $G(u, x, z)=\sum_{i=1}^{n-3} \pm z_{i}^{2} \pm x^{2}$ or $G(u, x, z)=\sum_{i=1}^{n-3} \pm z_{i}^{2} \pm x u \pm x^{3}$.

Thus for $s \in[0,1)$ we have:

$$
\left\{\begin{array}{l}
U(f(\phi(\theta(u, x, z), s)))=u+s A(u, x, z, s), \\
Y(f(\phi(\theta(u, x, z), s)))=G(u, x, z)+s B(u, x, z, s) .
\end{array}\right.
$$

Since we know that the rank of $T f$ at $P$ is 2 , we can write $B(u, x, z, s)$ as $b+C(u, x, z)+s D(u, x, z, s)$, where $b \neq 0$, and $C$ has no constant term. Further, since for $0 \leq|s|<\varepsilon$,

$$
\hat{f}(\psi(\theta(u, x, z), s))=f\left(\phi\left(\theta(u, x, z), s^{2}\right)\right),
$$

we have:

$$
\left\{\begin{array}{l}
U(\hat{f}(\psi(\theta(u, x, z), s)))=u+s^{2} A\left(u, x, z, s^{2}\right), \\
Y(\hat{f}(\psi(\theta(u, x, z), s)))=G(u, x, z)+s B(u, x, z, s) \\
\quad=G(u, x, z)+s^{2}(b+C(u, x, z))+s^{4} D\left(u, x, z, s^{2}\right) .
\end{array}\right.
$$

Since we need only keep track of the 3-jets, we can ignore the $s^{4}$-term completely, and keep only the linear parts of $A(u, x, z, 0)$ and of $C(u, x, z)$.

In the case that $G(u, x, z)=\mathscr{Q}(x, z)$, a nondegenerate quadratic form in $x$ and $z$, the normal form for $\hat{f}$ is determined by its 2 -jet and is therefore:

$$
\left\{\begin{array}{l}
U(\hat{f}(\psi(\theta(u, x, z), s)))=u \\
Y(\hat{f}(\psi(\theta(u, x, z), s)))=\mathscr{Q}(x, z)+s^{2} b .
\end{array}\right.
$$

In case $G(u, x, z)=\sum_{i=1}^{n-3} \pm z_{i}^{2} \pm x u \pm x^{3}$,

$$
\left\{\begin{aligned}
U(\hat{f}(\psi(\theta(u, x, z), s))) & =u+s^{2} A(u, x, z, 0), \\
Y(\hat{f}(\psi(\theta(u, x, z), s))) & =\sum_{i=1}^{n-3} \pm z_{i}^{2} \pm x u \pm x^{3}+s^{2}(b+C(u, x, z)) \\
& = \pm u x \pm x^{3}+\mathscr{Q}(z)+s^{2}(b+C(u, x, z)) .
\end{aligned}\right.
$$

It is an easy exercise to reduce this to the normal form, (2). If $A(u, x, z, 0)=$ $m u+M(x, z)+H(u, x, z)$, with $H$ of degree higher than 1 , in our computations we can ignore $H$ since, as we have already said, we ignore all terms in the coordinate expression for the mapping of degree greater than 3 . Changing coordinates as follows:

$$
\left\{\begin{array}{l}
\tilde{u}=u\left(1+m s^{2}\right)+s^{2} M(x, z), \\
\tilde{x}=x, \\
\tilde{z}=z, \\
\tilde{s}=s,
\end{array}\right.
$$


we obtain, dropping the 's:

$$
\left\{\begin{array}{l}
U(\hat{f}(\psi(\theta(u, x, z), s)))=u \\
Y(\hat{f}(\psi(\theta(u, x, z), s)))= \pm u x \pm x^{3}+\mathscr{Q}(z)+s^{2}(b+C(u, x, z)-x m) .
\end{array}\right.
$$

Now, letting $\tilde{s}=s \sqrt{\sigma(b+C(u, x, z)-x m)}$, where $b \sigma>0$, and leaving all other coordinates unchanged, gives us our desired normal form.

We now apply the theorem from [1] to our mapping, $\hat{f}$. We denote the components of $S(f)$ by $\left\{c_{i}\right\}$, oriented as required by the theorem as components of $S(\hat{f})$. We denote by $\left\{e_{k}\right\}$ the components of $\partial M$, if $\operatorname{dim} M=2$, and of $S(f \mid \partial M)$, if $\operatorname{dim} M>2$. Orienting the $\left\{e_{k}\right\}$ as components of $S(\hat{f})$, we obtain:

$$
\chi(\widehat{M})=2 \sum_{i} r\left(c_{i}\right)+\sum_{k} r\left(e_{k}\right)=2 \chi(M) .
$$

The reason that each $f\left(c_{i}\right)$ appears twice is that in $\widehat{M}, c_{i}$ appears as $c_{i} \times-1$ and $c_{i} \times 1$ however both of the copies of $c_{i}$ are oriented in the same way, hence both give equal contributions to $\chi(\widehat{M})$. Thus we have:

Theorem. Let $M$ be a compact, even-dimensional manifold with boundary, and let $f: M \rightarrow \mathbb{R}^{2}$ be a stable map, such that $S(f)$ does not meet $\partial M$. Let $\left\{c_{i}\right\}$ be the components of $S(f)$, and let $\left\{e_{k}\right\}$ be the components of $\partial M$ if $\operatorname{dim} M=2$ and of $S(f \mid \partial M)$ if $\operatorname{dim} M>2$. Then there is a unique orientation of the $\left\{c_{i}\right\}$ and the $\left\{e_{k}\right\}$, such that:

$$
\chi(M)=\sum_{i} r\left(c_{i}\right)+\frac{1}{2}\left(\sum_{k} r\left(e_{k}\right)\right)
$$

where $\chi(M)$ is the Euler characteristic of $M$ and $r\left(c_{i}\right)$ and $r\left(e_{k}\right)$ are the projectivized rotation numbers of $c_{i}$ and $e_{k}$, respectively.

\section{REFERENCES}

0. H. Levine, A. O. Petters, and J. Wambsganss, Applications of singularity theory to gravitational lensing I; Globally stable systems (in preparation).

1. H. Levine, Mappings of manifolds into the plane, Amer. J. Math. 88 (1966), 357-365.

2. M. Golubitsky and V. Guillemin, Stable mappings and their singularities, Springer, Berlin and New York, 1973.

3. René Thom, Les singularités des applications differentiables, Ann. Inst. Fourier (Grenoble) 4 (1955-1956), 43-87.

Department of Mathematics, Brandeis University, Waltham, Massachusetts 022549110

E-mail address: HLEVINE@BINAH. CC.BRANDEIS. EDU 